\title{
Preface to the Special Issue on Artificial Intelligence for Business Process Management 2018
}

\author{
Chiara Di Francescomarino ${ }^{1} \cdot$ Fabrizio Maria Maggi $^{2}$
}

Published online: 22 February 2020

c) Springer-Verlag GmbH Germany, part of Springer Nature 2020

The artificial intelligence (AI) discipline has been growing quickly in the last years by devising different approaches and being applied in a number of areas both in industry and academia. One of these areas is business process management (BPM). BPM provides novel application domains for AI. For instance, machine learning (ML) techniques have been used for mining event logs from different sourcesincluding sensor data, for predicting the future of ongoing execution traces, as well as for explaining deviant behaviors in business processes; planning approaches have been leveraged for modeling and analyzing business processes; constraint reasoning has been used as a key technology that underlies business rule engines. BPM and related fields can also influence the way AI develops. For instance, AI should be adapted to automate a manifold of business activities that today require qualified human workers; new research lines are required for adapting existing $\mathrm{AI}$ techniques to take into account the process dimension of BPM scenarios, such as the need for continuous predictions and recommendations over running process executions.

This Special Issue on Artificial Intelligence for Business Process Management 2018 of the Journal of Data Semantics focuses on the interplay between BPM and AI. It includes a keynote paper and the extended version of one selected paper from the International Workshop on Artificial Intelligence for Business Process Management (AI4BPM) 2018, which took place with the Business Process Management Conference (BPM) in September 2018, in Sydney, Australia. The invited papers were selected by taking into account the quality, significance and relevance of the results they present. All the papers went through an additional peer review process.
The paper "Declarative and Hybrid Process Discovery: Recent Advances and Open Challenges" authored by the keynote speaker, Dr. Slaats, focuses on the need for discovering concise models starting from highly varied behavior that often characterizes real-life processes. In this light, the work provides an overview of the recent advances in declarative and hybrid process discovery, discusses a number of open challenges that still remain, and proposes directions for future research.

The paper "Sensor-Based Human-Process Interaction in Discrete Manufacturing: Detection and Validation of Manual Assembly Tasks with Safe Flexibility" by Knoch et al. reports instead about the application of AI approaches for the automatic gathering of process-related sensor information. These fine-grained activities have then been used for the discovery of an assembly workflow, thus enabling the measurement of production time and quality, conformance checking and process optimization of manufacturing processes. The approach has been evaluated in a quantitative case study recording the assembly process in a laboratory setup.

The contributions to this Special Issue clearly show the strong interest of both research and industry in the interconnections between AI and BPM. These contributions also encourage the development of new approaches supporting this synergy and the definition of novel research directions able to deal with the different needs that could arise in this context.

Guest editors

Publisher's Note Springer Nature remains neutral with regard to jurisdictional claims in published maps and institutional affiliations.
$凶$ Chiara Di Francescomarino

dfmchiara@fbk.eu

Fabrizio Maria Maggi

f.m.maggi@ut.ee

1 Fondazione Bruno Kessler, Trento, Italy

2 University of Tartu, Tartu, Estonia 